\title{
Rendimiento de la canal y ganancia de peso en vacas de descarte con inducción del anestro por vías quirúrgica versus mecánica
}

\author{
Orquera, M.L. ${ }^{1}$; Pochon, D.O. ${ }^{2}$; Flores, S. ${ }^{1}$; Konrad, J.L. ${ }^{1}$; Crudeli, G.A. ${ }^{1}$ \\ Cátedras de Teriogenología ${ }^{1}$ y Bioestadística ${ }^{2}$, Facultad de Ciencias Veterinarias, Universidad Nacional \\ del Nordeste, Sargento Cabral 2139, Corrientes (3400), Argentina. Tel/fax: 03783-425753. \\ E-mail: gcrudeli@vet.unne.edu.ar.
}

\begin{abstract}
Resumen
Orquera, M.L.; Pochon, D.O.; Flores, S.; Konrad, J.L.; Crudeli, G.A.: Rendimiento de la canal y ganancia de peso en vacas de descarte con inducción de anestro por vías quirúrgica versus mecánica. Rev. vet. 22: 1, 64-67, 2011. El presente trabajo tuvo por objetivo evaluar la ganancia de peso y rendimiento de la canal en vacas descarte mestizas $(\mathrm{n}=47)$, con dispositivo intrauterino bovino (DIUB) y castradas quirúrgicamente. Con el propósito de adjudicar los tratamientos, las unidades experimentales fueron separadas al azar en tres grupos: G1) 15 vacas controles, G2) 19 vacas castradas y G3) 15 vacas con DIUB. Los animales fueron alojados en un potrero con pastura natural, con amplio predominio de Gatton panic. Se realizaron pesajes al inicio y cada treinta días, durante 120 días, para evaluar ganancia total de peso para los tres grupos. Al final los animales fueron faenados tras un ayuno previo de doce horas, a fin de determinar el rendimiento de la canal. Empleando un diseño completamente aleatorizado, el análisis de la variancia arrojó diferencias de peso significativas entre G1 y G3 (120 días): 77,6 vs 108 kg respectivamente $(\mathrm{p}<0,05)$ y entre G2 y G3 (120 días): 81,4 vs $108 \mathrm{~kg}$ respectivamente $(\mathrm{p}<0,05)$. También fueron significativas las diferencias del rendimiento de la canal entre G1 y G2 ( 51,02 vs $52,94 \%$ respectivamente, $\mathrm{p}<0,05)$, mientras que G2 y el G3 fueron similares para este parámetro. No se registraron diferencias significativas entre las ganancias de peso de G1 y G2 (120 días): 77,6 vs 81,4 kg. Los resultados demostraron que G3 fue más eficiente que G1 y G2 en ganancia de peso, en tanto que para el rendimiento de la canal G2 y G3 revelaron mejor desempeño. Se concluye que la aplicación de DIUB se traduce en una mejor relación costo-beneficio.
\end{abstract}

Palabras clave: vaca descarte, dispositivo intrauterino, castración quirúrgica, ganancia de peso, performance de canal.

\begin{abstract}
Orquera, M.L.; Pochon, D.O.; Flores, S.; Konrad, J.L.; Crudeli, G.A.: Carcass performance and weight gain in cull cows with anestrus induced by surgical versus mechanic ways. Rev. vet. 22: 1, 64-67, 2011. The purpose of this research is to assess the weight increment of crossbred cull cows with intra-uterine device (IUD) versus cows surgically neutered. To determine the efficacy of treatments, cows were grouped as follows: G1: 15 cows (control), G2: 19 cows neutered, and G3: 15 cows IUD. Animals were fed on natural pasture, being Gatton panic the most abundant. Weight controls were registered at the beginning of the assay, and then every thirty days during a period of 120 days. Finally, to register the carcass performance of the groups, cows were slaughtered with a previous fast of 12 hours. Significant liveweight differences were observed between G1 and G3 (120 days): 77.6 vs. 108 $\mathrm{kg}(\mathrm{p}<0.05)$, and between G2 and G3 (120 days): 81.4 vs. $108 \mathrm{~kg}(\mathrm{p}<0.05)$. Statistical differences in carcass performance were observed between G1 and G2: 51.02 vs. $52.94 \%(\mathrm{p}<0.05)$. No significant differences were observed between G1 and G2 (120 days): 77.6 vs. $81.4 \mathrm{~kg}$. Results indicate that animals from group G3 gained more weight in comparison to G1 and G2. Regarding carcass performance, groups G2 and G3 revealed a better efficiency. It can be concluded that the use of IUD leads to a better cost-benefit relationship.
\end{abstract}

Key words: cull cow, intra-uterine device, surgical neutering, weight gain, carcass performance. 


\section{INTRODUCCIÓN}

En Argentina la faena bovina durante el cuarto trimestre del año 2009 se ubicó levemente por encima de los cuatro millones de cabezas, cantidad moderadamente superior a los valores correspondientes al cuarto trimestre de $2008{ }^{10}$. La faena de hembras se ubicó a lo largo de 2009 en el $41,8 \%$ de la faena clasificada. Del total de hembras faenadas, alrededor del $30 \%$ fueron vacas, de las cuales la mitad se incluyó en la categoría vacas conserva ${ }^{8}$. Estos datos demuestran la importancia de la vaca de descarte en la producción de carne nacional.

Dentro de un sistema de cría bovina, la vaca refugo/ rechazo/descarte es un problema importante dentro de las empresas pecuarias de las regiones tradicionales de cría del país. En nuestra zona, debido al déficit invernal de los pastizales el vacuno pierde parte del peso obtenido durante el resto de las temporadas, es decir la estacionalidad del recurso forrajero es la principal limitante para el desarrollo de la ganadería regional. Debido a la necesidad de reducir la carga animal en los potreros, las vacas refugo que destetan su ternero, salen a la venta en junio-julio como categoría conserva, de menor precio en el mercado ${ }^{12}$.

El estro puede perjudicar el desempeño de las hembras en terminación, causando disturbios y estrés, afectando el consumo y la ganancia de peso de los animales ${ }^{9}$, así como aumentando la incidencia de carne dura, oscura y seca, generalmente con mayor número de hematomas ${ }^{2}$. Por ello han sido estudiadas distintas técnicas para disminuir la incidencia del estro y lograr una terminación más rápida de esos animales.

En razón del escaso número de trabajos que comparen formas de terminación de hembras de descarte para producción de carne en Argentina, en especial en la región nordeste, e intentando mejorar la rentabilidad de ese segmento de la producción pecuaria, el objetivo de este estudio fue evaluar el efecto de la inducción del anestro por vías mecánica (dispositivo intrauterino bovino DIUB) o quirúrgica (ovariectomía) sobre la ganancia de peso y rendimiento de la canal en vacas descarte.

\section{MATERIAL Y MÉTODOS}

Durante los meses comprendidos entre febrero y agosto, en un establecimiento ganadero de la localidad de Comandante Fontana (Formosa, Argentina), se utilizaron 49 vacas eliminadas del rodeo de cría (descarte). Los animales fueron objeto de examen clínico para excluir enfermedades; además se les practicó palpación rectal para evaluar el estado reproductivo y controlar el tamaño y funcionalidad de los ovarios, así como las condiciones del útero. Luego de identificadas, fueron distribuidas aleatoriamente en tres grupos, G1 $(n=15)$ : grupo control sin tratamiento, $G 2(n=19)$ : destinadas a castración quirúrgica y G3 $(\mathrm{n}=15)$ : consignadas a la aplicación del DIUB.
Entre los diferentes métodos quirúrgicos para realizar la ovariectomía, se optó por la técnica transvaginal de Dutto, por considerarla poco traumatizante, de bajo costo y pronta recuperación ${ }^{13}$. La colocación del DIUB fue realizada según lo describe la técnica ${ }^{5} \mathrm{y}$ con el tamaño de dispositivo adecuado ${ }^{14}$. Al comienzo de la experiencia las hembras fueron pesadas y recibieron tratamiento antiparasitario a base de ivermectina.

Fueron alimentadas por pastoreo directo con pastura natural a predominio de Gatton panic en estado de floración. Los grupos permanecieron mezclados en un potrero de 50 ha, durante un lapso de 120 días, sometidos a las mismas condiciones de manejo. La variable aumento de peso corporal se midió, previo ayuno de 12 horas, a partir del día de la castración hasta el final del ensayo (120 días).

De las 49 vacas que iniciaron el experimento, se llevaron a faena cuarenta y siete debido al deceso de dos animales castrados. Veinticuatro horas antes del sacrificio, tras ayuno de 12 horas, las vacas fueron pesadas para la obtención del valor pre-faena. Posteriormente se obtuvo el rendimiento de la canal, expresado en porcentaje del peso vivo previo a la faena.

Con los datos obtenidos, previa verificación de su normalidad, se efectuaron estadísticas descriptivas (media aritmética, desvío estándar y coeficiente de variación). A través de un diseño completamente aleatorizado se realizó análisis de la variancia, luego de verificar los supuestos de normalidad, homogeneidad de la variancia e independencia. Las comparaciones de medias se efectuaron por el test de Tuckey. Para todas las inferencias la hipótesis nula fue rechazada con un nivel de $\alpha=0,05$.

\section{RESULTADOS}

Ganancia de peso. Los pesos medios iniciales fueron de 315,341 y $350 \mathrm{~kg}$ para G1, G2 y G3 respectivamente. En la Tabla 1 se consignan las ganancias de peso de cada grupo, pudiendo observarse que no existieron diferencias significativas entre los grupos G1 (controles) y G2 (castrados), pero sí entre G3 (DIUB) versus $\mathrm{G} 1 / \mathrm{G} 2$.

Rendimiento de la canal. El mayor rendimiento fue obtenido por G2, seguido por G3. La menor performance correspondió a los controles (G1). Las diferencias fueron estadísticamente significativas sólo entre G1 y G2.

Tabla 1. Ganancias de peso y rendimiento de la canal en cada grupo.

\begin{tabular}{lccc}
\hline parámetro & G1 & G2 & G3 \\
$\begin{array}{l}\text { ganancia de peso } \\
\text { (kg totales) }\end{array}$ & $77,69 \pm 21,28^{\mathrm{a}}$ & $81,4 \pm 12,34^{\mathrm{a}}$ & $108,5 \pm 11,24^{\mathrm{b}}$ \\
\hline $\begin{array}{l}\text { rendimiento de } \\
\text { la canal (\%) }\end{array}$ & $51,02 \pm 0,93^{\mathrm{a}}$ & $52,94 \pm 1,49^{\mathrm{b}}$ & $51,88 \pm 2,34^{\mathrm{ab}}$ \\
\hline
\end{tabular}

En cada fila, letras distintas indican diferencias significativas $(\mathrm{p}<0,05)$. 
La diferencia de aumento de peso entre G3 y G1 (30 kg), implica una relación costo-beneficio positiva, pues al restarle el costo del dispositivo $(8 \mathrm{~kg})$, representa $22 \mathrm{~kg}$ de ventaja para las vacas con DIUB respecto a las que no lo tienen. La diferencia de rendimiento entre G3 y G1 fue de $0,86 \%$, lo que representa $4 \mathrm{~kg}$ más de peso vivo. El grupo G2 mostró una ganancia de peso de $3,71 \mathrm{~kg}$ más que G1, así como un rendimiento de $1,92 \%$, lo cual representaría $8 \mathrm{~kg}$ de peso vivo. El costo quirúrgico estaría representado por $10 \mathrm{~kg}$ de vaca gorda, dando como resultado un costo-beneficio negativo para la castración quirúrgica.

\section{DISCUSIÓN}

La ovariectomía suprime los estímulos hormonales para el inicio del ciclo estral y elimina la posibilidad de formación del cuerpo lúteo y de la síntesis de progesterona ${ }^{7}$. Además, las hembras castradas son más valorizadas pues ostentan un desarrollo considerablemente mayor de la porción anterior del cuerpo, tornándose más vistosas ${ }^{4}$. No obstante, la castración involucra cierto riesgo y representa un costo adicional en el proceso productivo ${ }^{13}$.

La ventaja de la ovariectomía reside en la posibilidad de evitar en el lote de vacas de invernada preñeces no deseadas, lo que facilita la eliminación de animales indeseables del rebaño y posibilita imprimir un desenvolvimiento más rápido de los terneros de última parición por el prolongamiento de la lactación. Además, confiere mayor practicidad en el manejo, pues hembras y machos pueden permanecer en la misma pastura o en confinamiento. De las técnicas quirúrgicas de castración de hembras bovinas, las más utilizadas son la que utiliza abordaje por el flanco y la castración vía vaginal, cada una con ventajas y desventajas ${ }^{13}$. En nuestro caso las complicaciones operatorias, llevaron al deceso de dos vacas a las dos semanas posteriores a la cirugía.

En discordancia con nuestros resultados, otros autores demostraron que vacas castradas mantenidas en pastura y confinamiento presentaban mayor ganancia diaria de peso que las no castradas ${ }^{1}$. En un trabajo se describe la menor deposición de gordura y reducción de la performance productiva y eficiencia alimentaria en hembras castradas, sugiriendo que la ausencia de estrógenos podría ser la responsable de estos cambios ${ }^{7}$.

Otra de las formas de suprimir el aparecimiento del estro, transformando así el efecto hormonal en factor de crecimiento y ganancia de peso, es la aplicación del DIUB. En las hembras rumiantes su aplicación afecta la periodicidad del estro y la función ovárica, disminuyendo la duración de la fase luteínica. Se asevera que el DIUB tiene acción anticonceptiva eficaz y promueve alteraciones en la función ovárica ${ }^{14}$. Su utilización inhibiría la ovulación de los animales y acarrearía bajos niveles de progesterona plasmática. El aumento de peso parecería estar relacionado con la elevación de la concentración de testosterona, ocasionando una hiperandrogenización de los animales y, posiblemente, una mayor actividad anabólica de las fibras musculares ${ }^{7}$. Asimismo el efecto económico del dispositivo anticonceptivo fue experimentado en vacas de descarte, donde las ganancias económicas, con y sin tratamiento, no fueron estadísticamente significativas ${ }^{3,6,11}$, contrariamente a los resultados aquí obtenidos.

En conclusión, los datos obtenidos indican que en las condiciones de manejo mencionadas y durante 120 días, la aplicación del DIUB promueve mayores ganancias de peso y mejor rendimiento de la canal que la castración quirúrgica.

\section{REFERENCIAS}

1. Chacur MG, De Faría MM, Oba E, Kronka S. 2007. Efeito da ovariectomia em novilhas Nelore x Angus, Bos taurus indicus x Bos taurus taurus sobre os ganhos de peso. Rev Ciências Agrárias (Brasil) 28: 317-322.

2. Crowe MA, Enricht WJ, Swift P. 1995. Growth and estrous behavior of heifers actively immunized against prostaglandin F2 $\alpha$. J Anim Sci 73: 345-352.

3. Fordyce G, Jubb TF, Fitzpatrick LA, White TR, Cooper NJ, Bolam MJ, Haddon DJ, Hill F, Docchio MJ. 2001. Contraceptive efficacy of an intra-uterine device in Brahman cattle. Anim Reprod Sci 65: 193-204.

4. Habermehl NL. 1993. Heifer ovariectomy using the Willis spay instrument: technique, morbidity and mortality. Can Vet J 34: 664-667.

5. Hafez ES. 2000. Reproducción e inseminación artificial en animales, McGraw-Hill, Cambridge, p. 26.

6. Machida Y, Sakaguchi I. 2003. Engorde semi-intensivo de vacas Nelore de descarte. Boletín Centro Tecnol Agropec JICA (Bolivia) 2: 41-43.

7. Márques JA, Prado IN, Zawadzki F. 2008. Os hormônios da reprodução e o desempenho de fêmeas. Rev Pub Vet (Brasil) 2: 6-19.

8. Oficina Nacional de Control Comercial Agropecuario (ONCCA). 2010. Sistemas de precios bovinos, República Argentina, Informe 888. www.oncca.gov.ar.

9. Prado IN, Martins AS, Alcalde CR. 2000. Desempenho de novilhas alimentadas com rações contendo milho ou casca de mandioca como fonte energética e farelo de algodão ou levedura como fonte protéica. Rev Bras Zoot 29 : 278-287.

10. Rearte D. 2005. Debate sobre el stock de vientres. Diario La Nación, Suplem. "El Campo", Buenos Aires (Argentina), febr 26, p. 1-2.

11. Restle J, Roso C, De Oliveira AN, Alves DC, Pascoal LL, Pereira JR. 2000. Suplementação energética para vacas de descarte de diferentes idades em terminação em pastagem cultivada de estação fria sob pastejo horario. Rev Bras Zoot 29: 1216-1222.

12. Revidatti MA, Capellari A, Coppo NB, Coppo JA, Fioranelli SA, Navamuel M. 2000. Utilización de residuos cítricos en la suplementación de vacas de invernada. Evaluación de las ganancias de peso y condición corporal. Anales Reunión Comunic Científ \& Tecnol UNNE (Corrientes, Argentina), V-031. 
13. Silva LA, Almeida CF, Viana PR, Cordeiro AC, Rabelo RE, Eurides D, Fioravanti MC. 2004. Descrição de duas técnicas cirúrgicas para castração de fêmeas bovinas. Ciencia Anim Bras 5: 47-53.

14. Turin EM. 1997. Effects of a copper-bearing intrauterine device on the ovarian function, body weight gain and pregnancy rate of nulliparous heifers. Theriogenology: 47: 1327-1336. 